\title{
Towards a Theory of Chaos Explained as Travel on Riemann Surfaces
}

\author{
F Calogero ${ }^{1,2}$, D Gómez-Ullate ${ }^{3}, \mathbf{P}$ M Santini ${ }^{1,2}$, and $\mathbf{M}$ \\ Sommacal $^{4,5}$ \\ ${ }^{1}$ Dipartimento di Fisica, Università di Roma "La Sapienza", Roma, Italy. \\ 2 Istituto Nazionale di Fisica Nucleare, Sezione di Roma, Italy. \\ 3 Departamento de Física Teórica II, Universidad Complutense, Madrid, Spain. \\ ${ }^{4}$ Dipartimento di Matematica e Informatica, Università degli Studi di Perugia, \\ Perugia, Italy. \\ 5 Istituto Nazionale di Fisica Nucleare, Sezione di Perugia, Italy. \\ E-mail: francesco.calogero@roma1.infn.it, david.gomez-ullate@fis.ucm.es, \\ paolo.santini@roma1.infn.it, matteo.sommacal@pg.infn.it
}

\begin{abstract}
This paper presents a more complete version than hitherto published of our explanation of a transition from regular to irregular motions and more generally of the nature of a certain kind of deterministic chaos. To this end we introduced a simple model analogous to a three-body problem in the plane, whose general solution is obtained via quadratures all performed in terms of elementary functions. For some values of the coupling constants the system is isochronous and explicit formulas for the period of the solutions can be given. For other values, the motions are confined but feature aperiodic (in some sense chaotic) motions.

This rich phenomenology can be understood in remarkable, quantitative detail in terms of travel on a certain (circular) path on the Riemann surfaces defined by the solutions of a related model considered as functions of a complex time. This model is meant to provide a paradigmatic first step towards a somewhat novel understanding of a certain kind of chaotic phenomena.
\end{abstract}

PACS numbers: 05.45-a, 02.30.Hq, 02.30.Ik. 
Towards a Theory of Chaos Explained as Travel on Riemann Surfaces

\section{Introduction}

The fact that the distinction among integrable or nonintegrable behaviors of a dynamical system is somehow connected with the analytic structure of the solutions of the model under consideration as functions of the independent variable "time" (considered as a complex variable) is by no means a novel notion. It goes back to classical work by Carl Jacobi, Henri Poincaré, Sophia Kowalevskaya, Paul Painlevé and others. In recent times some of us had the good fortune to hear in several occasions such ideas clearly described by Martin Kruskal 22, 23. A simple-minded rendition of his teachings can be described as follows: for an evolution to be integrable, it should be expressible, at least in principle, via formulas that are not excessively multivalued in terms of the dependent variable, entailing that, to the extent this evolution is expressible by analytic functions of the dependent variable (considered as a complex variable), it might possess branch points, but it should not feature an infinity of them that is dense in the complex plane of the independent variable.

A number of techniques collectively known as Painlevé analysis have been resurrected and further developed over the last few decades (for a review see, for instance, [28]). In essence, they consider an ansatz of the local behaviour of a solution near a singularity in terms of a Laurent series, introducing it in the equations and determining the leading orders and resonances (terms in the expansion at which arbitrary constants appear). Painlevé analysis has been extended to test for the presence of algebraic branching (weak Painlevé property [28]) by considering a Puiseaux series instead of a Laurent series. These analytic techniques (which have been algorithmized and are now available in computer packages) constitute a useful tool in the investigation of integrability: in many nonlinear systems where no solution in closed form is known, Painlevé analysis provides information on the type of branching featured by the general solution or by special classes of solutions. It has also proved useful to identify special values of the parameters for which generally chaotic systems such as Hénon-Heiles or Lorenz are integrable [4, 16].

On the opposite side of the spectrum lie chaotic dynamical systems and it is natural to investigate the singularity structure of their solutions. Tabor and his collaborators initiated this study in the early eighties for the Lorenz system [29] and the Henon-Heiles Hamiltonian [16]. They realized that the singularities of the solutions in complex-time are important for the real-time evolution of the system. The complex time analytic structure was studied by extensions of the Painlevé analysis involving the introduction of logarithmic terms in the expansion - the so called $\Psi$-series which provides a local representation of the solutions in the neighbourhood of a singularity in the chaotic regime. Their local analytic approach was complemented by numerical techniques developed for finding the location of the singularities in complex time and determining the order of branching [14. In all the chaotic systems under study, they observed numerically that the singularities in complex time cluster on a natural boundary with self-similar structure [15. An analytic argument to explain the mechanism that leads to recursive singularity clustering was given in 24. Similar studies relating singularity structure, chaos and integrability have been performed by Bountis and his collaborators. Going beyond the local techniques described above, the emphasis is put on a global property of the solutions: whether their Riemann surface has a finite or an infinite number of sheets. Bountis proposes to use the term integrable for the first case and non-integrable for the second 2, 3. . Using mostly numerical evidence he conjectures that in the non-integrable cases the Riemann surfaces are 
infinitely-sheeted and the projection on the complex plane of the singularities is dense. Combining analytical and numerical results for a simple ODE, Bountis and Fokas [18] have identified chaotic systems with the property that the singularities of their solutions are dense.

Painlevé analysis and its extensions are useful and widely applicable. However, as local techniques, they provide no information on the global properties of the Riemann surfaces of the solutions, such as: the number and location of the movable branch points a solution has, and moreover how the different sheets of the Riemann surface are connected together at those branch points. Understanding these global properties is important for the dynamics; a detailed analysis of the Riemann surface associated to the solutions of a dynamical system, whenever it can be done, provides a much deeper understanding than can be obtained by local techniques alone.

This is precisely the motivation of the investigation reported herein: to introduce and study a model which is simple enough that a full description of its Riemann surface can be performed, yet complicated enough to feature a rich behaviour, possibly including irregular or chaotic characteristics.

Such a model was initially presented in 1 and in this paper we continue investigating its properties. This line of research originates from a "trick" that is convenient to identify isochronous systems [7, 9] - a change of dependent and independent variables, with the new independent variable traveling on a path in the complex plane. Later it was shown that many isochronous systems can be written by a suitable modification of a large class of complex ODEs [10, 9]. Using local analysis and numerical integration in two many-body systems in the plane [13, 11], it was discovered that outside the isochrony region there exist periodic solutions with much higher periods as well as possibly aperiodic solutions, and the connection among this phenomenology and the analytic structure of the corresponding solutions as functions of complex time was illuminated. However, those systems were too complicated for a complete description of the Riemann surface to be achieved.

Recent work along these lines includes problems whose solution is obtained by inversion of a hyperelliptic integral: the corresponding Riemann surfaces have been studied in [17, 19], together with the implications on the dynamical properties of the models.

In the present paper we provide many details that were reported without proof in [1, such as the description of the general solution by quadratures, and we also exhibit other properties of the model that were not present in [1, such as similarity solutions, equilibrium configurations and small oscillations. Our investigation of this model will continue in a subsequent publication, [12, where the full description of the geometrical properties of the Riemann surface will be given.

This paper is organized as follows: in Section 2 we present our model, including in particular the relationship among its physical version (independent variable: the real time $t$ ) and its auxiliary version (independent variable: a complex variable $\tau$ ), and we outline the main findings reported in this paper. In Section 3 we discuss the equilibrium configurations of our physical model, and the behavior of this system in the neighborhood of these solutions, and we also obtain certain exact similarity solutions of our model and discuss their stability. In Section 4 we discuss the analytic structure of the solutions of the auxiliary model via local analyses à la Painlevé, since the analytic structure of these solutions plays a crucial role in determining the time evolution of our physical model. In Section 5 we show how the general solution of our model can be achieved by quadratures and in Section 6 we outline the behavior of 
our model based on these results. Finally, in Section 7 we summarize our results and comment on future developments. This paper also contains a few Appendices, where certain calculations are confined (to avoid interrupting inconveniently the flow of the presentation) as well as certain additional findings.

\section{Presentation of the model}

In this section we introduce the model treated in this paper, and we outline our main findings that are then proven and further discussed in subsequent sections.

\subsection{The auxiliary model}

The auxiliary model on which we focus in this paper is characterized by the following system of three coupled nonlinear ODEs:

$$
\zeta_{n}^{\prime}=\frac{g_{n+2}}{\zeta_{n}-\zeta_{n+1}}+\frac{g_{n+1}}{\zeta_{n}-\zeta_{n+2}} .
$$

Notation: here and hereafter indices such as $n, m$ range from 1 to 3 and are defined $\bmod (3) ; \tau$ is the (complex) independent variable of this auxiliary model; the three functions $\zeta_{n} \equiv \zeta_{n}(\tau)$ are the dependent variables of this auxiliary model, and we assume them to be as well complex; an appended prime always denotes differentiation with respect to the argument of the functions it is appended to (here, of course, with respect to the complex variable $\tau$ ); and the three quantities $g_{n}$ are arbitrary "coupling constants" (possibly also complex; but in this paper we restrict consideration mainly to the case with real coupling constants; this is in particular hereafter assumed in this section). In the following we will often focus on the "semisymmetrical case" characterized by the equality of two of the three coupling constants, say

$$
g_{1}=g_{2}=g, \quad g_{3}=f,
$$

since in this case the treatment is simpler yet still adequate to exhibit most aspects of the phenomenology we are interested in. More special cases are the "fully symmetrical", or "integrable", one characterized by the equality of all three coupling constants,

$$
g=f, \quad g_{1}=g_{2}=g_{3}=g,
$$

and the "two-body" one, with only one nonvanishing coupling constant, say

$$
g_{1}=g_{2}=g=0, g_{3}=f \neq 0 .
$$

In this latter case clearly

$$
\zeta_{3}^{\prime}=0, \quad \zeta_{3}(\tau)=\zeta_{3}(0)
$$

(see (10) and the remaining two-body problem is trivially solvable,

$$
\zeta_{s}(\tau)=\frac{1}{2}\left[\zeta_{1}(0)+\zeta_{2}(0)\right]-(-)^{s}\left\{\frac{1}{4}\left[\zeta_{1}(0)-\zeta_{2}(0)\right]^{2}+f \tau\right\}^{1 / 2}, s=1,2,
$$

while the justification for labeling the fully symmetrical case (3) as "integrable" will be clear from the following (or see Section 2.3.4.1 of [8]). Before introducing our physical model, let us note that the auxiliary system (11) is invariant under translations of both the independent variable $\tau$ (indeed, it is autonomous) and the dependent variables $\zeta_{n}(\tau)\left(\zeta_{n}(\tau) \Rightarrow \zeta_{n}(\tau)+\zeta_{0}, \zeta_{0}^{\prime}=0\right)$, and it is moreover invariant under an appropriate simultaneous rescaling of the independent and the dependent variables. 


\subsection{The trick and the physical model}

The trick mentioned above, relating the auxiliary model to the physical model, amounts in our present case to the introduction of the (real) independent variable $t$ ("physical time"), as well as the three (complex) dependent variables $z_{n} \equiv z_{n}(t)$, via the following positions:

$$
\begin{aligned}
& \tau=\frac{\exp (2 i \omega t)-1}{2 i \omega}, \\
& z_{n}(t)=\exp (-i \omega t) \zeta_{n}(\tau) .
\end{aligned}
$$

We hereafter assume the constant $\omega$ to be real (for definiteness, positive, $\omega>0$; note that for $\omega=0$ the change of variables disappears), and we associate to it the period

$$
T=\frac{\pi}{\omega}
$$

Note that this change of variables entails that the initial values $z_{n}(0)$ of the "particle coordinates" $z_{n}(t)$ coincide with the initial values $\zeta_{n}(0)$ of the dependent variables of the auxiliary model (1):

$$
z_{n}(0)=\zeta_{n}(0) \text {. }
$$

It is easily seen that, via this change of variables, (5), the equations of motion (11) satisfied by the quantities $\zeta_{n}(\tau)$ entail the following (autonomous) equations of motion (in the real time $t$ ) for the particle coordinates $z_{n}(t)$ :

$$
\dot{z}_{n}+i \omega z_{n}=\frac{g_{n+2}}{z_{n}-z_{n+1}}+\frac{g_{n+1}}{z_{n}-z_{n+2}} .
$$

Here and hereafter superimposed dots indicate differentiations with respect to the time $t$.

So, this model (7) describes the "physical evolution" which we study. Note that its equations of motion, (7), are of Aristotelian, rather than Newtonian, type: the "velocities" $\dot{z}_{n}$, rather than the "accelerations" $\ddot{z}_{n}$, of the moving particles are determined by the "forces". In Appendix D we discuss the connection of this model with more classical many-body problems, characterized by Newtonian equations of motion.

Let us immediately emphasize two important qualitative aspects of the dynamics of our physical model (77). The "one-body force" represented by the second term, $i \omega z_{n}$, in the left-hand side of the equations of motion (7) becomes dominant with respect to the "two-body forces" appearing in the right-hand side in determining the dynamics whenever the (complex) coordinate $z_{n}$ of the $n$-th particle becomes large (in modulus). Hence when $\left|z_{n}(t)\right|$ is very large, the solution $z_{n}(t)$ of (7) is approximated by the solution of $\dot{z}_{n}+i \omega z_{n} \approx 0$ implying that $z_{n}(t)$ is characterized by the behavior $z_{n}(t) \approx c \exp (-i \omega t)$, therefore the trajectory of the $n$-th particle tends to rotate (clockwise, with period $2 T$ ) on a (large) circle. This effect causes all motions of our physical model, (7), to be confined. Secondly, it is clear that the two-body forces (see the right-hand side of (7)) cause a singularity whenever there is a collision of two (or all three) of the particles as they move in the complex $z$-plane, and become dominant whenever two or three particles get very close to each other, namely in the case of near collisions. But if the three particles move aperiodically in a confined region (near the origin) of the complex z-plane, a lot of near collisions shall indeed occur. And since the outcome of a near collision is likely to be quite different depending on which 
side two particles scatter past each other - and this, especially in the case of very close near collisions, depends sensitively on the initial data of the trajectory under consideration - we see here a mechanism complicating the motion, indeed causing some kind of chaos associated with a sensitive dependence of the motion on its initial data. This suggests that our model (7), in spite of its simplicity, is likely to be rich enough to cause an interesting dynamical evolution. We will see that this is indeed the case. But before proceeding with this investigation let us interject two remarks (somewhat related to each other).

Remark 1 This system (7) is still invariant under translations of the independent variable $t$ (indeed, it is again autonomous) but, in contrast to (1), it is no longer invariant under translations of the dependent variables $z_{n}(t)$ nor under a simple rescaling of the independent variable $t$ and of the dependent variables $z_{n}(t)$.

Remark 2 The general solution of the equations of motion (7) has the structure

$$
z_{n}(t)=z_{C M}(t)+\check{z}_{n}(t),
$$

where the three functions $\check{z}_{n}(t)$ satisfy themselves the same equations of motion (7) as well as the additional restriction

$$
\check{z}_{1}(t)+\check{z}_{2}(t)+\check{z}_{3}(t)=0
$$

which is clearly compatible with these equations of motion, and correspondingly $z_{C M}(t)$ is the center of mass of the system (7),

$$
z_{C M}(t)=\frac{z_{1}(t)+z_{2}(t)+z_{3}(t)}{3}
$$

and it evolves according to the simple formula

$$
z_{C M}(t)=z_{C M}(0) \exp (-i \omega t)=Z \exp (-i \omega t) .
$$

In Section 3 (and Appendix A) we determine the equilibrium configurations of our physical model, namely the values $z_{n}^{(\mathrm{eq})}$ of the three particle coordinates $z_{n}$ such that

$$
z_{n}=z_{n}^{(\mathrm{eq})}, \quad \dot{z}_{n}=0
$$

satisfy the equations of motion (7), and we ascertain the behavior of our system in the neighborhood of these configurations. In the second part of Section 3, and then almost always in the rest of this paper (and throughout the rest of this section) we restrict for simplicity consideration to the semisymmetrical case, see (2). A main finding in Section 3 (and Appendix A) is that in the semisymmetrical case our model (77) possesses generally two equilibrium configurations $z_{n}^{(\text {eq) }}$. We moreover determine the three exponents $\gamma^{(m)}$ characterizing the small oscillations of our system in the neighborhood of each of these two configurations, defined according to the standard formulas (see Section 3 )

$$
\begin{aligned}
& z_{n}(t)=z_{n}^{(\mathrm{eq})}+\varepsilon w_{n}(t), \\
& w_{n}^{(m)}(t)=\exp \left(-i \gamma^{(m)} \omega t\right) v_{n}^{(m)},
\end{aligned}
$$

where of course $\varepsilon$ is an infinitesimally small parameter and the quantities $v_{n}^{(m)}$ are time-independent. We find that the first two of these three exponents take in both cases the simple values

$$
\gamma^{(1)}=1, \quad \gamma^{(2)}=2 ;
$$


the first of these corresponds of course to the center-of-mass motion, see (9b). As for the third exponent $\gamma^{(3)}$, we find for one equilibrium configuration

$$
\gamma^{(3)}=\frac{f+8 g}{f+2 g}=\frac{1}{\mu}
$$

and for the other

$$
\gamma^{(3)}=\frac{f+8 g}{3 g}=\frac{2}{1-\mu} .
$$

Here we have introduced the constant $\mu$,

$$
\mu=\frac{f+2 g}{f+8 g}
$$

whose value, as we shall see, plays an important role in determining the dynamical evolution of our model: in particular, this evolution does largely depend on whether or not $\mu$ is a real rational number, and if it is rational,

$$
\mu=\frac{p}{q}
$$

with $p$ and $q$ coprime integers (and $q$ positive, $q>0$ ), on whether the two natural numbers $|p|$ and $q$ are large or small. A hint of this is already apparent from the results we just reported: while the solutions $w_{n}^{(1)}(t)$ and $w_{n}^{(2)}(t)$, see (11), are both periodic with period $2 T$ (see (5c); in fact $w_{n}^{(2)}(t)$ is periodic with period $T$ ), the solution $w_{n}^{(3)}(t)$, see $(11)$, is periodic with the period $\tilde{T}$,

$$
\tilde{T}=\frac{2 T}{\gamma^{(3)}}
$$

which is clearly congruent to Tonly if $\mu$ is rational, see (15) and (13) - implying then that the small oscillations around the equilibrium configurations are always completely periodic with a period which is a finite integer multiple of $T$.

In Section 3 we also introduce the special class of (exact and completely explicit) "similarity" solutions of our equations of motion, (7), and analyze their stability, namely the solutions of our system in the immediate neighborhood of these similarity solutions.

\subsection{Conserved quantities}

It is important to note at this point that the auxiliary model (1) possesses conserved quantities, which will be used in Section 5 to obtain its general solution by quadratures. Firstly, due to the translational invariance it is obvious that the quantity

$$
Z=\frac{1}{3} \sum_{n=1}^{3} \zeta_{n}
$$

does not depend on $\tau$. In addition, the analysis of Section 5 shows that in the semisymmetrical case there exists an extra conserved quantity given by

$$
\tilde{K}=\left(2 \zeta_{3}-\zeta_{1}-\zeta_{2}\right)^{-2}\left[1-\frac{\left(\zeta_{1}-\zeta_{2}\right)^{2}+\left(\zeta_{2}-\zeta_{3}\right)^{2}+\left(\zeta_{3}-\zeta_{1}\right)^{2}}{2 \mu\left(2 \zeta_{3}-\zeta_{1}-\zeta_{2}\right)^{2}}\right]^{\mu-1} .
$$

Here the constant $\mu$ is defined in terms of the coupling constants $g$ and $f$, see (2), by (14). We already mentioned that the value of this parameter (in particular, whether 
or not $\mu$ is a rational number) plays an important role in determining the dynamical evolution of our model. A hint of this is now provided by the appearance of this number $\mu$ as an exponent in the right-hand side of (18), since this exponent characterizes the multivaluedness of the dependence of the constant $\tilde{K}$ on the coordinates $\zeta_{n}$.

\section{Equilibrium configurations, small oscillations and similarity solutions of the physical model}

In this section we discuss, firstly, the equilibrium configurations of our physical model, (7), and its behavior near equilibrium, and secondly, a special, explicit "similarity" solution of our model and its stability.

The equilibrium configurations of our physical model (7),

$$
z_{n}(t)=z_{n}^{(\mathrm{eq})}, \quad \dot{z}_{n}(t)=0,
$$

(see (10)) are clearly characterized by the algebraic equations

$$
i \omega z_{n}^{(\mathrm{eq})}=\frac{g_{n+1}}{z_{n}^{(\mathrm{eq})}-z_{n+2}^{(\mathrm{eq})}}+\frac{g_{n+2}}{z_{n}^{(\mathrm{eq})}-z_{n+1}^{(\mathrm{eq})}} .
$$

These algebraic equations entail

$$
z_{1}^{\text {(eq) }}+z_{2}^{(\mathrm{eq})}+z_{3}^{(\mathrm{eq})}=0 .
$$

It is now convenient to set

$$
z_{n}^{(\mathrm{eq})}=(2 i \omega)^{-1 / 2} \alpha_{n},
$$

so that the equilibrium equations (20) read as follows:

$$
\frac{\alpha_{n}}{2}=\frac{g_{n+1}}{\alpha_{n}-\alpha_{n+2}}+\frac{g_{n+2}}{\alpha_{n}-\alpha_{n+1}} .
$$

These algebraic equations can be conveniently (see below) rewritten as follows:

$$
\alpha_{n}=\beta_{n+1}\left(\alpha_{n}-\alpha_{n+2}\right)+\beta_{n+2}\left(\alpha_{n}-\alpha_{n+1}\right),
$$

via the position

$$
\beta_{n}=\frac{2 g_{n}}{\left(\alpha_{n-1}-\alpha_{n+1}\right)^{2}} .
$$

We now note that, in order that the three equations (24a) (which are linear in the three unknowns $\alpha_{n}$, although only apparently so, see (24b) ) have a nonvanishing solution, the quantities $\beta_{n}$ must cause the following determinant to vanish:

$$
\left|\begin{array}{ccc}
\beta_{2}+\beta_{3}-1 & -\beta_{3} & -\beta_{2} \\
-\beta_{3} & \beta_{3}+\beta_{1}-1 & -\beta_{1} \\
-\beta_{2} & -\beta_{1} & \beta_{1}+\beta_{2}-1
\end{array}\right|=0 .
$$

To analyze the small oscillations of our system (7) around its equilibrium configurations we now set

$$
z_{n}(t)=z_{n}^{(\mathrm{eq})}+\varepsilon w_{n}(t),
$$

(see (11a)) and we then get (linearizing by treating $\varepsilon$ as an infinitesimally small parameter)

$$
\dot{w}_{n}+i \omega w_{n}+i \omega \beta_{n+1}\left(w_{n}-w_{n+2}\right)+\beta_{n+2}\left(w_{n}-w_{n+1}\right)=0 .
$$


Therefore the three exponents $\gamma^{(m)}$ characterizing the small oscillations around equilibrium via the formula

$$
w_{n}^{(m)}(t)=\exp \left(-i \gamma^{(m)} \omega t\right) v_{n}^{(m)},
$$

providing three independent solutions of the system of linear ODEs (26b), are the three eigenvalues of the symmetrical matrix

$$
\mathbf{B}=\left(\begin{array}{ccc}
\beta_{2}+\beta_{3}+1 & -\beta_{3} & -\beta_{2} \\
-\beta_{3} & \beta_{3}+\beta_{1}+1 & -\beta_{1} \\
-\beta_{2} & -\beta_{1} & \beta_{1}+\beta_{2}+1
\end{array}\right),
$$

and the three 3 -vectors $\vec{v}^{(m)} \equiv\left(v_{1}^{(m)}, v_{2}^{(m)}, v_{3}^{(m)}\right)$ are the corresponding eigenvectors,

$$
\sum_{\ell=1}^{3} B_{n \ell} v_{\ell}^{(m)}=\gamma^{(m)} v_{n}^{(m)}
$$

Hence the three exponents $\gamma^{(m)}$ are the three roots of the "secular equation"

$$
\left|\begin{array}{ccc}
\beta_{2}+\beta_{3}+1-\gamma & -\beta_{3} & -\beta_{2} \\
-\beta_{3} & \beta_{3}+\beta_{1}+1-\gamma & -\beta_{1} \\
-\beta_{2} & -\beta_{1} & \beta_{1}+\beta_{2}+1-\gamma
\end{array}\right|=0 .
$$

Clearly these three roots are given by the following formulas:

$$
\gamma^{(1)}=1, \quad \gamma^{(2)}=2, \quad \gamma^{(3)}=2\left(\beta_{1}+\beta_{2}+\beta_{3}\right) .
$$

Indeed the determinant (30) vanishes for $\gamma=\gamma^{(1)}=1$ (when each line sums to zero) and for $\gamma=\gamma^{(2)}=2$ (see (25)), and the third solution,

$$
\gamma^{(3)}=2\left(\beta_{1}+\beta_{2}+\beta_{3}\right),
$$

is then implied by the trace condition

$$
\text { trace }[\mathbf{B}]=3+2\left(\beta_{1}+\beta_{2}+\beta_{3}\right)=\gamma^{(1)}+\gamma^{(2)}+\gamma^{(3)} .
$$

The first of these 3 solutions, $\gamma^{(1)}=1$, corresponds to the center of mass motion (it clearly entails $v_{n}^{(1)}=v^{(1)}$, see (27) and (28)).

In the semisymmetrical case (22) the equations (23) (or equivalently (24)) characterizing, via (22), the equilibrium configurations can be solved explicitly (see Appendix A). One finds that there are two distinct equilibrium configurations (in fact four, if one takes account of the trivial possibility to exchange the roles of the two "equal" particles with labels 1 and 2), the first of which reads simply

$$
z_{3}^{(\mathrm{eq})}=0, \quad z_{1}^{(\mathrm{eq})}=-z_{2}^{(\mathrm{eq})}=z^{(\mathrm{eq})}, \quad\left(z^{(\mathrm{eq})}\right)^{2}=\frac{f+2 g}{2 i \omega},
$$

while the second has a slightly more complicated expression (see Appendix A). Note however that, in both cases, there holds the relation

$$
\left(z_{1}^{(\mathrm{eq})}-z_{2}^{(\mathrm{eq})}\right)^{2}+\left(z_{2}^{(\mathrm{eq})}-z_{3}^{(\mathrm{eq})}\right)^{2}+\left(z_{3}^{(\mathrm{eq})}-z_{1}^{(\mathrm{eq})}\right)^{2}=\frac{3(f+2 g)}{i \omega} .
$$

Moreover, in both cases the corresponding values for the eigenvalue $\gamma^{(3)}$, see (32), are easily evaluated. The first solution yields (see (13a)

$$
\gamma^{(3)}=\frac{f+8 g}{f+2 g}=\frac{1}{\mu}=\frac{q}{p},
$$


where, for future reference, we expressed $\gamma^{(3)}$ not only in terms of the parameter $\mu$, see (14), but as well in terms of its rational expression (15) (whenever applicable), while the second solution likewise yields

$$
\gamma^{(3)}=\frac{f+8 g}{3 g}=\frac{2}{1-\mu}=\frac{2 q}{q-p} .
$$

Note that this implies that in the "integrable" case (3) both these formulas, (36a) and $(36 b)$, yield $\gamma^{(3)}=3$; but it is easily seen that in this case only the first equilibrium configuration (34) actually exists. So in the "integrable" case the oscillations around the (only) equilibrium configuration (34) are the linear superposition of three periodic motions (see (27)) with respective periods $2 T, T$ and $\frac{2 T}{3}$ (see (5c)). Also in the "two-body" case (4) the second equilibrium configuration does not exist, while the first formula, (36a), yields $\gamma^{(3)}=1$, so in this case the small oscillations around the (only) equilibrium configuration (34) are the linear superposition of two periodic motions, with periods $2 T$ and $T$ (see (5c); consistently with the explicit solution, easily obtainable from (4c) via (5)).

As can be easily verified, the equilibrium configurations (19) with (20) are merely the special case corresponding to $z_{C M}(0)=0, c=0$ of the following two-parameter family of (exact) "similarity" solutions of our equations of motion (7):

$$
\begin{aligned}
& z_{n}(t)=z_{C M}(t)+\tilde{z}_{n}(t ; c), \\
& \tilde{z}_{n}(t ; c) \equiv z_{n}^{(\text {eq })}[1+c \exp (-2 i \omega t)]^{1 / 2},
\end{aligned}
$$

with the center of mass coordinate $z_{C M}(t)$ evolving according to (9b). The two arbitrary (complex) constants featured by this solution are of course $z_{C M}(0)=Z$ (see (9b)) and $c$, while the constants $z_{n}^{\text {(eq) }}$ 's are defined as in the preceding section, see (20).

Clearly these (exact) solutions correspond, via the trick (5), the relation (22) (which is clearly consistent with (23) and (20)) and the simple relation

$$
\tau_{b}=\frac{c-1}{2 i \omega}
$$

to the two-parameter family

$$
\zeta_{n}(\tau)=Z+\alpha_{n}\left(\tau-\tau_{b}\right)^{1 / 2},
$$

of (exact) solutions of (1).

Let us now discuss the stability of this solution, (37b). To this end we set

$$
z_{n}(t)=\tilde{z}_{n}(t ; c)+\varepsilon \tilde{w}_{n}(t)
$$

and we insert this ansatz in our equations of motion (7), linearizing them by treating $\varepsilon$ as an infinitesimally small parameter. We thus get

$$
\dot{\tilde{w}}_{n}+i \omega \tilde{w}_{n}+\frac{i \omega\left[\beta_{n+1}\left(\tilde{w}_{n}-\tilde{w}_{n+2}\right)+\beta_{n+2}\left(\tilde{w}_{n}-\tilde{w}_{n+1}\right)\right]}{1+c \exp (-2 i \omega t)}=0,
$$

having used the definition (24b). Clearly the solution of this system of ODEs reads

$$
\tilde{w}_{n}(t)=\exp (-i \omega t) \chi_{n}(\vartheta) \text {, }
$$

with

$$
\vartheta \equiv \vartheta(t)=t-(2 i \omega)^{-1} \log \left[\frac{1+c \exp (-2 i \omega t)}{1+c}\right]
$$


and the functions $\chi_{n}(\vartheta)$ solutions of the autonomous linear system of first-order ODEs

$$
\chi_{n}^{\prime}+i \omega\left[\beta_{n+1}\left(\chi_{n}-\chi_{n+2}\right)+\beta_{n+2}\left(\chi_{n}-\chi_{n+1}\right)\right]=0,
$$

where the primes denote of course differentiation with respect to $\vartheta$. Hence (see (26b) ) the three independent solutions of this linear system are

$$
\chi_{n}^{(m)}(\vartheta)=\exp (i \omega \vartheta) w_{n}^{(m)}(\vartheta),
$$

with the functions $w_{n}^{(m)}$ defined by (27) (of course with $t$ replaced by $\vartheta$ ), yielding via (27) and (41a) with (41b) the following two equivalent expressions for the three independent solutions of the linear system (40b):

$$
\begin{aligned}
& \tilde{w}_{n}^{(m)}(t)=\left[\frac{1+c \exp (-2 i \omega t)}{1+c}\right]^{\left(\gamma^{(m)}-1\right) / 2} \exp \left(i \gamma^{(m)} \omega t\right) \tilde{v}_{n}^{(m)}, \\
& \tilde{w}_{n}^{(m)}(t)=\left[\frac{\exp (2 i \omega t)+c}{1+c}\right]^{\left(\gamma^{(m)}-1\right) / 2} \exp (i \omega t) \tilde{v}_{n}^{(m)}
\end{aligned}
$$

Here the three exponents $\gamma^{(m)}$ are defined as above, see (31), and likewise the "eigenvectors" $\tilde{v}_{n}^{(m)}$ coincide with those defined above up to (arbitrary) normalization constants $c^{(m)}$,

$$
\tilde{v}_{n}^{(m)}=c^{(m)} v_{n}^{(m)} .
$$

Note the equivalence of the two expressions (42a) and (42b) (the motivation for writing these two versions of the same formula will be immediately clear).

For $m=1,2,3$ these solutions, see (42b), are periodic functions of the (real) time $t$ with period $2 T$ if $|c|>1$. If instead $|c|<1$, the solutions (see (42a)) with $m=1$ respectively $m=2$ are periodic with periods $2 T$ respectively $T$ (see (31)). The solution with $m=3$ is periodic if $\gamma^{(3)}$ is real, but with the period $\frac{2 T}{\left|\gamma^{(3)}\right|}$ which is not congruent to $T$ if $\gamma^{(3)}$ is irrational; it grows exponentially with increasing time if $\operatorname{Im}\left[\gamma^{(3)}\right]<0$, implying instability of the solution (37) in this case, and it instead decays exponentially if $\operatorname{Im}\left[\gamma^{(3)}\right]>0$, implying a limit cycle behavior in configuration space, namely asymptotic approach to a solution completely periodic with period $T$ or $2 T$ depending whether the center of mass of the system is fixed at the origin or itself moving with period $2 T$; but note that in this paper we restrict our attention to the case with real coupling constants.

\section{Analytic structure of the solutions of the auxiliary model}

In this section we discuss the properties of analyticity as functions of the complex variable $\tau$ of the solutions $\zeta_{n}(\tau)$ of the auxiliary model (1) (with arbitrary values of the 3 coupling constants $g_{n}$, i. e. not restricted by the semisymmetrical condition (2): except when this is explicitly specified, see below). In particular we show first of all that, for appropriate initial data characterized by sufficiently large values of the moduli of all three interparticle distances, namely by the condition (see (66) ) that the quantity

$$
\zeta_{\min }=\min _{n, m=1,2,3 ; n \neq m}\left|\zeta_{n}(0)-\zeta_{m}(0)\right|
$$


be adequately large, the solutions $\zeta_{n}(\tau)$ are holomorphic in a disk $D_{0}$ of (arbitrarily large) radius $d_{0}$ centered at the origin, $\tau=0$, of the complex $\tau$-plane (of course the "adequately large" value of the quantity $\zeta_{\min }$ depends on $d_{0}$, and on the magnitude of the three coupling constants $g_{n}$; see (51) below). We moreover discuss via a local analysis a la Painlevé the nature of the singularities of the solutions $\zeta_{n}(\tau)$ of the auxiliary model (1D) as functions of the complex variable $\tau$ and we thereby justify the assertions made in this respect in Section 2.

To prove the first point, set

$$
\sigma_{n}(\tau)=\zeta_{n}(\tau)-\zeta_{n}(0),
$$

so that these quantities $\sigma_{n}(\tau)$ vanish initially,

$$
\sigma_{n}(0)=0,
$$

and, as a consequence of (1), satisfy the equations of motion

$$
\begin{aligned}
\sigma_{n}^{\prime}(\tau)= & \frac{g_{n+1}}{\zeta_{n}(0)-\zeta_{n+2}(0)+\sigma_{n}(\tau)-\sigma_{n+2}(\tau)} \\
& +\frac{g_{n+2}}{\zeta_{n}(0)-\zeta_{n+1}(0)+\sigma_{n}(\tau)-\sigma_{n+1}(\tau)} .
\end{aligned}
$$

A standard theorem (see, for instance, [20]) guarantees then that these quantities $\sigma_{n}(\tau)$ - hence as well the functions $\zeta_{n}(\tau)$, see (44a) - are holomorphic in $\tau$ (at least) in a disk $D_{0}$ centered at the origin $\tau=0$ in the complex $\tau$-plane, the radius $d_{0}$ of which is bounded below by the inequality

$$
d_{0}>\frac{b}{4 M}
$$

(this formula coincides with the last equation of Section 13.21 of [20, with the assignments $m=3$ and $a=\infty$, the first of which is justified by the fact that the system (44c) features 3 coupled equations, the second of which is justified by the autonomous character of the equations of motion (44C)). The two positive quantities $b$ and $M$ in the right-hand side of this inequality are defined as follows. The quantity $b$ is required to guarantee that the right-hand sides of the equations of motion (44c) be holomorphic (as functions of the dependent variables $\sigma_{n}$ ) provided these quantities satisfy the three inequalities

$$
\left|\sigma_{n}\right| \leq b ;
$$

clearly in our case a sufficient condition to guarantee this is provided by the single restriction

$$
b<\frac{\zeta_{\min }}{2},
$$

with $\zeta_{\text {min }}$ defined by (43). The second quantity in the right-hand side of (45), $M \equiv M(b)$, is the upper bound of the right-hand sides of (44c) when the quantities $\sigma_{n}$ satisfy the inequality (46); but of course the inequality (45) holds a fortiori if we overestimate $M$, as we shall presently do. Indeed clearly the equations of motion (44C) with (46) and (47) entail

$$
M<\frac{4 G}{\zeta_{\min }-2 b},
$$

with

$$
G=\max _{n=1,2,3}\left|g_{n}\right| .
$$


Insertion of (48) in (45) yields

$$
d_{0}>\frac{b\left(\zeta_{\min }-2 b\right)}{16 G}
$$

hence, setting $b=\frac{\zeta_{\min }}{4}$ (to maximize the right-hand side; note the consistency of this assignment with (47)),

$$
d_{0}>\frac{\zeta_{\min }^{2}}{128 G},
$$

confirming the assertion made above (that $d_{0}$ can be made arbitrarily large by choosing $\zeta_{\min }$ adequately large).

Next, let us show, via a local analysis à la Painlevé, that the singularities as functions of the complex variable $\tau$ of the general solutions $\zeta_{n}(\tau)$ of our auxiliary model (1) associated with a coincidence of two of the three components $\zeta_{n}$ are squareroot branch points (recall that a singularity at finite $\tau$ of a solution $\zeta_{n}(\tau)$ of the evolution equations (1) may only occur when the right-hand side of these equations diverges). Such a singularity occurs for those values $\tau_{b}$ of the independent variable $\tau$ such that two of the three functions $\zeta_{n}$ coincide, for instance

$$
\zeta_{1}\left(\tau_{b}\right)=\zeta_{2}\left(\tau_{b}\right) \neq \zeta_{3}\left(\tau_{b}\right) .
$$

The square-root character of these branch points is evident from the following ansatz characterizing the behavior of the solutions of (1) in the neighborhood of these singularities:

$$
\begin{aligned}
& \zeta_{s}(\tau)=\zeta_{b}-(-1)^{s} \alpha\left(\tau-\tau_{b}\right)^{1 / 2}+v_{s}\left(\tau-\tau_{b}\right)+\sum_{k=3}^{\infty} \alpha_{s}^{(k)}\left(\tau-\tau_{b}\right)^{k / 2}, \quad s=1,2 \\
& \zeta_{3}(\tau)=\zeta_{3 b}+v_{3}\left(\tau-\tau_{b}\right)+\sum_{k=3}^{\infty} \alpha_{3}^{(k)}\left(\tau-\tau_{b}\right)^{k / 2}
\end{aligned}
$$

with

$$
\alpha^{2}=g_{3}, \quad v_{3}=-\frac{g_{1}+g_{2}}{\zeta_{b}-\zeta_{3 b}}, \quad v_{s}=\frac{g_{s}+5 g_{s+1}}{6\left(\zeta_{b}-\zeta_{3 b}\right)}, \quad s=1,2 \bmod (2)
$$

and the constants $\alpha_{n}^{(k)}$ determinable (in principle) recursively (for $k=3,4, \ldots$ ) by inserting this ansatz in (1), so that, to begin with

$$
\begin{aligned}
\alpha_{3}^{(3)} & =\frac{2 \alpha\left(g_{2}-g_{1}\right)}{3\left(\zeta_{b}-\zeta_{3}\right)^{2}} \\
\alpha_{s}^{(3)} & =-(-1)^{s} \frac{\alpha}{36\left(\zeta_{b}-\zeta_{3}\right)^{2}}\left[3\left(g_{s}-7 g_{s+1}\right)+\frac{\left(g_{1}-g_{2}\right)^{2}}{g_{3}}\right], \\
s & =1,2 \bmod (2),
\end{aligned}
$$

and so on. The diligent reader will verify the consistency of this procedure, for any assignment of the three constants $\tau_{b}, \zeta_{b}$, and $\zeta_{3 b}$, which remain undetermined except for the obvious restrictions $\tau_{b} \neq 0, \zeta_{b} \neq 0, \zeta_{3 b} \neq \zeta_{b}$. The fact that (53) contains three arbitrary (complex) constants - the maximal number of integration constants compatible with the system of three first-order ODEs (1) - shows that this ansatz is indeed adequate to represent locally, in the neighborhood of its singularities occurring at $\tau=\tau_{b}$, the general solution of (11). 
An analogous analysis of the behavior of the solutions of the system (1) near the values of the independent variable $\tau$ where a triple coincidence of all three functions $\zeta_{n}$ occurs (corresponding to the excluded assignment $\zeta_{3 b}=\zeta_{b}$ in the above ansatz (53)), indicates, somewhat surprisingly, that such a triple coincidence,

$$
\zeta_{1}\left(\tau_{b}\right)=\zeta_{2}\left(\tau_{b}\right)=\zeta_{3}\left(\tau_{b}\right)=Z
$$

might also occur for the general solution of the system (11). This conclusion is reached via a local analysis analogous to that performed above, and is then confirmed (for the semisymmetrical case, see (2) ) by the exact treatment of Section 5. Indeed the natural extension of the above ansatz (53) characterizing the behavior of the solutions of (11) in the neighborhood of such singularities, corresponding to a triple coincidence, see (54), of the three functions $\zeta_{n}(\tau)$, reads as follows:

$$
\zeta_{n}(\tau)=Z+\eta_{n}\left(\tau-\tau_{b}\right)^{(1-\gamma) / 2}+\alpha_{n}\left(\tau-\tau_{b}\right)^{1 / 2}+\mathrm{o}\left(\left|\tau-\tau_{b}\right|^{1 / 2}\right)
$$

provided

$$
\operatorname{Re}(\gamma)<0 .
$$

Here the three constants $\alpha_{n}$ are determined, as can be easily verified, just by the three nonlinear algebraic equations (23) that were found in the preceding section while investigating the equilibrium configurations of our physical system (7), while the three constants $\eta_{n}$, as well as the exponent $\gamma$, are required to satisfy the algebraic equations

$$
\frac{(\gamma-1) \eta_{n}}{2}=\frac{g_{n+1}\left(\eta_{n}-\eta_{n+2}\right)}{\left(\alpha_{n}-\alpha_{n+2}\right)^{2}}+\frac{g_{n+2}\left(\eta_{n}-\eta_{n+1}\right)}{\left(\alpha_{n}-\alpha_{n+1}\right)^{2}}
$$

These algebraic equations, (23) and (56), can be conveniently rewritten as follows:

$$
\begin{aligned}
& \alpha_{n}=\beta_{n+1}\left(\alpha_{n}-\alpha_{n+2}\right)+\beta_{n+2}\left(\alpha_{n}-\alpha_{n+1}\right), \\
& (\gamma-1) \eta_{n}=\beta_{n+1}\left(\eta_{n}-\eta_{n+2}\right)+\beta_{n+2}\left(\eta_{n}-\eta_{n+1}\right),
\end{aligned}
$$

via the introduction of the quantities $\beta_{n}$, see (24b). Note that in this manner these two sets of equations, (57a) and (57b), have a quite similar look, which should however not mislead the reader to underestimate their basic difference: the three equations (57a) are merely a convenient way to rewrite, via the definition (24b), the three nonlinear equations (23), which determine (albeit not uniquely, see Appendix A) the three constants $\alpha_{n}$; while the equations (57b) are three linear equations for the three quantities $\eta_{n}$, hence they can determine these three unknowns only up to a common multiplicative constant (provided they admit a nontrivial solution: see below).

Of course these linear equations (57b) admit the trivial solution $\eta_{n}=0$, and it is easily seen that there indeed is a special (exact) solution of the equations of motion (11) having this property, see (39) with the constants $\alpha_{n}$ determined by (23) and computed, for the semisymmetrical model, in Appendix A. This "similarity solution" (39) of the system (1) has been discussed in the preceding section; but let us emphasize here that it only provides a two-parameter $\left(Z\right.$ and $\left.\tau_{b}\right)$ class of solutions of the equations of motion (1), while the general solution of this system of three first-order ODEs must of course feature three arbitrary parameters.

A general solution of the evolution equations (1) corresponds instead to the ansatz (55a) if the linear equations (57b) for the three coefficients $\eta_{n}$ admit a nonvanishing solution, because in such a case, as mentioned above, a common scaling parameter for these three coefficients remains as an additional (third) free parameter (besides $Z$ and $\tau_{b}$ ). The condition for this to happen is the vanishing of the determinant of 
the coefficients of these three linear equations, (57b), namely again validity of the determinantal condition (30), a cubic equation for the unknown $\gamma$, which determines, as discussed in the preceding section, the three values (31) of this quantity. But the first two of these values, $\gamma=\gamma^{(1)}=1$ and $\gamma=\gamma^{(2)}=2$ (see (31)), are not consistent with the requirement (55b). The third solution, $\gamma=\gamma^{(3)}=2\left(\beta_{1}+\beta_{2}+\beta_{3}\right)$ (see (31) ) might instead be consistent with the requirement (55b), and whenever this happens the ansatz (55) indicates that the general solution of the system of ODEs (11) does feature a "triple coincidence", see (54), and identifies the character of the corresponding branch point.

In the semisymmetrical case (2) the equations characterizing the equilibrium configuration, (23) or equivalently (57), can be solved (see Appendix A). One finds that there are two distinct solutions of these nonlinear equations (23) (in fact four, since each solution has a trivial counterpart obtained by exchanging the role of the two "equal" particles with labels 1 and 2). The first solution yields for $\gamma=\gamma^{(3)}$ the value (36a), which is consistent with the condition (55b) iff

$$
\operatorname{Re}(\mu)<0 \text {, }
$$

and it yields for the branch point exponent, see (55a) , the value

$$
\frac{1-\gamma}{2}=\frac{\mu-1}{2 \mu}=\frac{p-q}{2 p} ;
$$

while the second solution yields for $\gamma^{(3)}$ the value (36b), which is consistent with the condition (55b) iff

$$
\operatorname{Re}(\mu)>1 \text {, }
$$

and it yields for the branch point exponent, see (55a), the value

$$
\frac{1-\gamma}{2}=\frac{\mu+1}{2(\mu-1)}=\frac{p+q}{2(p-q)} .
$$

The last equality in (59) and (61) are of course only valid if $\mu$ is rational, $\mu=p / q$.

Note that these findings imply that the branch point associated with "triple coincidences" is not (only) of square-root type, being also characterized, see (55a), by the exponent $\frac{1-\gamma}{2}$, the value of which depends on the value of the parameter $\mu$, see (59) and (61); however this kind of branch point is not present if

$$
0<\operatorname{Re}(\mu)<1,
$$

since in this case neither (58) nor (60) are satisfied.

The results presented in this section are not entirely rigorous, since the local analysis of the singularities we performed above on the basis of appropriate ansätze should be complemented by proofs that the relevant expansions converge. Moreover these analyses provide information on the nature of the branch points, but neither on their number nor their location. But these results are confirmed and complemented below (see Section 5) by the analysis of the exact general solution of the equations of motion (11). Our motivation for having nevertheless presented here a discussion of the character of the singularities of the solutions of (1) via a local analysis à la Painlevé is because an analogous treatment may be applicable to models which are not as explicitly solvable as that treated in this paper (see for instance [13] and [11). 
Towards a Theory of Chaos Explained as Travel on Riemann Surfaces

\section{General solution by quadratures}

In this section we obtain and discuss the general solutions of our models, (11) and (7).

But since the general solution of the physical model (7) is easily obtained via the trick

(5) from the general solution of the auxiliary problem (1), we focus to begin with on this model.

A first constant of the motion is provided by the center-of-mass coordinate

$$
Z=\frac{\zeta_{1}+\zeta_{2}+\zeta_{3}}{3}
$$

since the equations of motion (11) clearly entail

$$
Z^{\prime}=0
$$

hence

$$
Z(\tau)=Z(0)
$$

And clearly the general solution of (1) reads

$$
\zeta_{n}(\tau)=Z+\check{\zeta}_{n}(\tau)
$$

with the set of 3 functions $\check{\zeta}_{n}(\tau)$ providing themselves a solution of (1), independent of the value of $Z$ and satisfying the (compatible) constraint

$$
\check{\zeta}_{1}(\tau)+\check{\zeta}_{2}(\tau)+\check{\zeta}_{3}(\tau)=0 .
$$

It is moreover clear that the equations of motion (1) entail

$$
\zeta_{1}^{\prime} \zeta_{1}+\zeta_{2}^{\prime} \zeta_{2}+\zeta_{3}^{\prime} \zeta_{3}=g_{1}+g_{2}+g_{3}
$$

hence there also holds the relation

$$
\zeta_{1}^{2}+\zeta_{2}^{2}+\zeta_{3}^{2}=2\left(g_{1}+g_{2}+g_{3}\right)\left(\tau-\tau_{0}\right) .
$$

It is now convenient to set, as in the Appendix B of [13,

$$
\begin{aligned}
& \zeta_{s}=Z-\left(\frac{2}{3}\right)^{1 / 2} \rho \cos \left[\theta-(-1)^{s} \frac{2 \pi}{3}\right], \quad s=1,2, \\
& \zeta_{3}=Z-\left(\frac{2}{3}\right)^{1 / 2} \rho \cos \theta
\end{aligned}
$$

Then, summing the squares of these three formulas and using the identities

$$
\begin{aligned}
& \cos (\theta)+\cos \left(\theta+\frac{2 \pi}{3}\right)+\cos \left(\theta-\frac{2 \pi}{3}\right)=0, \\
& \cos ^{2}(\theta)+\cos ^{2}\left(\theta+\frac{2 \pi}{3}\right)+\cos ^{2}\left(\theta-\frac{2 \pi}{3}\right)=\frac{3}{2},
\end{aligned}
$$

one easily gets

$$
\zeta_{1}^{2}+\zeta_{2}^{2}+\zeta_{3}^{2}=3 Z^{2}+\rho^{2}
$$

or equivalently

$$
\rho^{2}=\frac{1}{3}\left[\left(\zeta_{1}-\zeta_{2}\right)^{2}+\left(\zeta_{2}-\zeta_{3}\right)^{2}+\left(\zeta_{3}-\zeta_{1}\right)^{2}\right]
$$

hence, from 6656 ,

$$
\rho^{2}=2\left(g_{1}+g_{2}+g_{3}\right)\left(\tau-\tau_{0}\right)-3 Z^{2}=2\left(g_{1}+g_{2}+g_{3}\right)\left(\tau-\tau_{1}\right),
$$


Towards a Theory of Chaos Explained as Travel on Riemann Surfaces

$$
\tau_{1}=\tau_{0}+\frac{3 Z^{2}}{2\left(g_{1}+g_{2}+g_{3}\right)},
$$

which also entails

$$
\rho^{\prime} \rho=g_{1}+g_{2}+g_{3} .
$$

Here we assume that the sum of the three coupling constants $g_{n}$ does not vanish, $g_{1}+g_{2}+g_{3} \neq 0$. The special case in which this sum does instead vanish is treated in Appendix C. The expression of the constant $\tau_{1}$ in terms of the initial data is of course $($ see $(\underline{69 c}))$

$$
\tau_{1}=-\frac{\rho^{2}(0)}{2\left(g_{1}+g_{2}+g_{3}\right)}
$$

namely (see (69c))

$$
\tau_{1}=-\frac{\zeta_{1}^{2}(0)+\zeta_{2}^{2}(0)+\zeta_{3}^{2}(0)-3 Z^{2}}{2\left(g_{1}+g_{2}+g_{3}\right)}
$$

or equivalently (see $(\underline{69 d})$

$$
\tau_{1}=-\frac{\left(\zeta_{1}-\zeta_{2}\right)^{2}+\left(\zeta_{2}-\zeta_{3}\right)^{2}+\left(\zeta_{3}-\zeta_{1}\right)^{2}}{6\left(g_{1}+g_{2}+g_{3}\right)} .
$$

There remains to compute $\theta(\tau)$, or rather

$$
u(\tau)=\cos \theta(\tau) .
$$

Inserting the ansatz (66) in the equation of motion (11) with $n=3$, one easily gets

$$
\begin{aligned}
\rho^{2}(\cos \theta)^{\prime}\left(4 \cos ^{2} \theta-1\right)= & \left(4 g_{1}+4 g_{2}+g_{3}\right) \cos \theta-4\left(g_{1}+g_{2}+g_{3}\right) \cos ^{3} \theta \\
& +\sqrt{3}\left(g_{1}-g_{2}\right) \sin \theta .
\end{aligned}
$$

From now on in this section - for simplicity, and because it is sufficient for our purposes - we restrict attention to the semisymmetrical case (2), so that the last equation becomes simply, via (71),

$$
\rho^{2} u^{\prime}\left(4 u^{2}-1\right)=(f+8 g) u-4(f+2 g) u^{3} .
$$

The general case without the restriction (2) is treated in Appendix C.

This ODE can be easily integrated via a quadrature (using (69c)), and this leads to the following formula:

$$
[u(\tau)]^{-2 \mu}\left[u^{2}(\tau)-\frac{1}{4 \mu}\right]^{\mu-1}=K\left(\tau-\tau_{1}\right),
$$

where the parameter $\mu$ is defined by (14) and $K$ is an integration constant. Here we are of course assuming that $f+8 g \neq 0$ (see (14)); the case when this does not happen is treated in Appendix C. (Also recall that, as promised above, we shall treat in Appendix $\mathrm{C}$ the case in which the sum of the three coupling constants $g_{n}$ vanishes, namely when $f+2 g=0$, which entails $\mu=0$, see (14)). As for the quantity $K$ in $(74)$, it is an (a priori arbitrary) integration constant. It is a matter of elementary algebra to express this constant in terms of the original dependent variables $\zeta_{n}$ (via (74), (69c), (71) and (66)), and one thereby obtains the relation

$$
K=12(f+2 g) \tilde{K}
$$

with $\tilde{K}$ defined by (18). This finding justifies the assertion that $\tilde{K}$ is a constant of motion, see Section 2.3; and of course it determines the value to be assigned to 
the constant $K$ in the context of the initial-value problem. Likewise the value to be assigned, in the context of the initial-value problem, to the constant $\tau_{1}$ appearing in the right-hand side of (74) is given by the formula

$$
K \tau_{1}=-[u(0)]^{-2 \mu}\left[u^{2}(0)-\frac{1}{4 \mu}\right]^{\mu-1},
$$

where (see (71) and $(\underline{66 b})$ )

$$
u(0)=-\left(\frac{3}{2}\right)^{1 / 2} \frac{\zeta_{3}(0)-Z}{\rho(0)}
$$

namely

$$
u(0)=-\frac{2 \zeta_{3}(0)-\zeta_{1}(0)-\zeta_{2}(0)}{\left[2\left\{\left[\zeta_{1}(0)-\zeta_{2}(0)\right]^{2}+\left[\zeta_{2}(0)-\zeta_{3}(0)\right]^{2}+\left[\zeta_{3}(0)-\zeta_{1}(0)\right]^{2}\right\}\right]^{1 / 2}} .
$$

Of course in these formulas the initial values $\zeta_{n}(0)$ of the coordinates $\zeta_{n}(\tau)$ of the auxiliary problem (11) can be replaced by the initial values $z_{n}(0)$ of the physical problem (7), see (6).

Let us emphasize that we have now reduced, via (66) with (69c) and (71), the solution of our problem (11) to the investigation of the function $u(\tau)$ of the complex variable $\tau$, defined for $\tau \neq 0$ as the solution of the (nondifferential) equation (74) that evolves by continuity from $u(0)$ at $\tau=0$.

To proceed with our analysis an additional change of variables is now convenient. We introduce the new (complex) independent variable $\xi$ by setting

$$
\xi=\frac{K\left(\tau-\tau_{1}\right)}{4 \mu},
$$

and the new (complex) dependent variable $w \equiv w(\xi)$ by setting

$$
w(\xi)=4 \mu[u(\tau)]^{2} .
$$

Thereby the expression of the solution (66) of our original problem (11) reads

$$
\begin{aligned}
\zeta_{s}(\tau) & =Z-\left(\frac{f+2 g}{3 K}\right)^{1 / 2} \xi^{1 / 2}\left\{-[w(\xi)]^{1 / 2}+(-)^{s}[12 \mu-3 w(\xi)]^{1 / 2}\right\}, \\
s & =1,2 \\
\zeta_{3}(\tau) & =Z-2\left(\frac{f+2 g}{3 K}\right)^{1 / 2}[\xi w(\xi)]^{1 / 2},
\end{aligned}
$$

while the (nondifferential) equation that determines the dependence of $w(\xi)$ on the (complex) variable $\xi$ reads

$$
[w(\xi)-1]^{\mu-1}[w(\xi)]^{-\mu}=\xi .
$$

Note that this equation is independent of the initial data; it only features the constant $\mu$, which only depends on the coupling constants, see (14).

We conclude that the solution of our physical problem (7) as the real time variable $t$ evolves onwards from $t=0$ is essentially given, via (80) and (5), by the evolution of the solution $w(\xi)$ of this (nondifferential) equation, (81), as the complex variable $\xi$ travels round and round on the circle $\Xi$ in the complex $\xi$-plane defined by the equation (see (78) and (5a))

$$
\xi=R \exp (2 i \omega t)+\bar{\xi}=R[\exp (2 i \omega t)+\eta],
$$


namely on the circle with center $\bar{\xi}$ and radius $|R|$. The parameters $R$ and $\bar{\xi}$ (or $\eta$ ) depend on the initial data according to the formulas (implied by (78), (5), (75), (18), (70c))

$$
\begin{aligned}
R & =\frac{3(f+8 g)}{2 i \omega\left[2 z_{3}(0)-z_{1}(0)-z_{2}(0)\right]^{2}}[1-\kappa]^{\mu-1}, \\
\bar{\xi} & =R \eta, \\
\eta & =\frac{i \omega\left\{\left[\zeta_{1}(0)-\zeta_{2}(0)\right]^{2}+\left[\zeta_{2}(0)-\zeta_{3}(0)\right]^{2}+\left[\zeta_{3}(0)-\zeta_{1}(0)\right]^{2}\right\}}{3(f+2 g)}-1, \\
\kappa & =\frac{2 \mu\left[2 \zeta_{3}(0)-\zeta_{1}(0)-\zeta_{2}(0)\right]^{2}}{\left[\zeta_{1}(0)-\zeta_{2}(0)\right]^{2}+\left[\zeta_{2}(0)-\zeta_{3}(0)\right]^{2}+\left[\zeta_{3}(0)-\zeta_{1}(0)\right]^{2}} .
\end{aligned}
$$

Of course in these formulas the initial values $\zeta_{n}(0)$ of the coordinates $\zeta_{n}(\tau)$ of the auxiliary problem (11) can be replaced by the initial values $z_{n}(0)$ of the coordinates $z_{n}(t)$ of the physical problem (7), see (6).

Let us emphasize that, as the complex variable $\xi$ travels on the circle $\Xi$-taking the time $T$ to make each round, see (82a) and (5c) - the dependent variable $w(\xi)$ travels on the Riemann surface determined by its dependence on the complex variable $\xi$, as entailed by the equation (81) that relates $w(\xi)$ to its argument $\xi$ - starting at $t=0$ from $\xi=\xi_{0}$,

$$
\begin{aligned}
\xi_{0} & =\bar{\xi}+R=(\eta+1) R, \\
\xi_{0} & =\frac{i \omega R\left\{\left[\zeta_{1}(0)-\zeta_{2}(0)\right]^{2}+\left[\zeta_{2}(0)-\zeta_{3}(0)\right]^{2}+\left[\zeta_{3}(0)-\zeta_{1}(0)\right]^{2}\right\}}{3(f+2 g)}
\end{aligned}
$$

(see (82)) and correspondingly from $w\left(\xi_{0}\right)=w_{0}$,

$$
w_{0}=\frac{1}{\kappa}=\frac{\left[\zeta_{1}(0)-\zeta_{2}(0)\right]^{2}+\left[\zeta_{2}(0)-\zeta_{3}(0)\right]^{2}+\left[\zeta_{3}(0)-\zeta_{1}(0)\right]^{2}}{2 \mu\left[2 \zeta_{3}(0)-\zeta_{1}(0)-\zeta_{2}(0)\right]^{2}}
$$

$($ see $(82 e)$ ).

Let us therefore now discuss the structure of this Riemann surface, namely the analytic properties of the function $w(\xi)$ defined by (81). There are two types of singularities, the "fixed" ones occurring at values of the independent variable $\xi$, and correspondingly of the dependent variable $w$, that can be read directly from the structure of the equation (81) under investigation, and the "movable" ones (this name being given to underline their difference from the fixed ones) occurring at values of the independent and dependent variables, $\xi$ and $w$, that cannot be directly read from the structure of the equation (81) under investigation (they "move" as the initial data are modified).

\subsection{Movable singularities}

To investigate their nature it is convenient to differentiate (81), obtaining thereby (using again (81))

$$
\xi w^{\prime}=-\frac{w(w-1)}{w-\mu}
$$


where the prime indicates of course differentiation with respect to $\xi$. (Note that this ODE is implied by the nondifferential equation (81), while its solution reproduces the nondifferential equation (81) up to multiplication of its right-hand side by an arbitrary constant). The position of the singularities, $\xi_{b}$, and the corresponding values of the dependent variable, $w_{b} \equiv w\left(\xi_{b}\right)$, are then characterized by the vanishing of the denominator in the right-hand side of this formula, yielding the relation

$$
w_{b}=\mu,
$$

which, combined with (81) (at $\xi=\xi_{b}$ ) is easily seen to yield

$$
\begin{aligned}
& \xi_{b}=\xi_{b}^{(k)}=r \exp (2 \pi i \mu k), \quad k=1,2,3, \ldots, \\
& \xi_{b}=\xi_{b}^{(k)}=r \exp \left[i \frac{2 \pi p k}{q}\right], \quad k=1,2, \ldots, q, \\
& r=(\mu-1)^{-1}\left(\frac{\mu-1}{\mu}\right)^{\mu} .
\end{aligned}
$$

In the last, $87 \mathrm{87c}$ ), of these formulas it is understood that the principal determination is to be taken of the $\mu$-th power appearing in the right-hand side. The first of these formulas, (87a), shows clearly that the number of these branch points is infinite if the parameter $\mu$ is irrational, and that they then sit densely on the circle $B$ in the complex $\xi$-plane centered at the origin and having radius $r$, see (87c). Note that this entails that the generic point on the circle $B$ is not a branch point (just as a generic real number is not rational); but every generic point on the circle $B$ has some branch point (in fact, an infinity of branch points!) arbitrarily close to it (just as every generic real number has an infinity of rational numbers arbitrarily close to it). As for the second of this formulas, (87b), it is instead appropriate to the case in which the parameter $\mu$ is rational, see (15), in which case the branch points sit again on the circle $B$ in the complex $\xi$-plane, but there are only a finite number, $q$, of them.

These singularities are all square root branch points, as implied by the following standard proof. Set, for $\xi \approx \xi_{b}$,

$$
w(\xi)=\mu+a\left(\xi-\xi_{b}\right)^{\beta}+o\left(\left|\xi-\xi_{b}\right|^{\operatorname{Re}(\beta)}\right),
$$

with the assumption (immediately verified, see below) that

$$
0<\operatorname{Re}(\beta)<1 \text {. }
$$

It is then immediately seen that the insertion of this ansatz in (85) (is consistent and) yields

$$
\beta=\frac{1}{2}, \quad a^{2}=\frac{2(1-\mu)}{\xi_{b}}=-2\left(\frac{\mu}{\mu-1}\right)^{\mu} .
$$

Note that these results confirm the treatment of Section 4 the square root branch points of $w(\xi)$ identified here, see (86), are easily seen to correspond, via (80), to the pair coincidence $\zeta_{1}\left(\tau_{b}\right)=\zeta_{3}\left(\tau_{b}\right)$ or $\zeta_{2}\left(\tau_{b}\right)=\zeta_{3}\left(\tau_{b}\right)$; while there is an additional class of square-root branch points which only affect $\zeta_{1}(\tau)$ and $\zeta_{2}(\tau)$, but neither $\zeta_{3}(\tau)$ nor $w(\xi)$, and occur at

$$
w=4 \mu
$$


due to the vanishing of the second square-root term inside the curly bracket in the right-hand side of (80a), and correspond therefore to the coincidence $\zeta_{1}\left(\tau_{b}\right)=\zeta_{2}\left(\tau_{b}\right)$. The corresponding values of $\xi$ (as implied by (89a) with (81)) are

$$
\xi=\frac{(4 \mu-1)^{\mu-1}}{(4 \mu)^{\mu}}=\frac{1}{4 \mu}\left(1-\frac{1}{4 \mu}\right)^{\mu-1}
$$

(we use the plural to refer to these values because of the multivaluedness of the function in the right-hand side of this formula).

\subsection{Fixed singularities}

Next, let us consider the "fixed" singularities, which clearly can only occur at $\xi=\infty$ and at $\xi=0$, with corresponding values for $w$.

Let us investigate firstly the nature of the singularities at $\xi=\infty$. Two behaviors of $w(\xi)$ are then possible for $\xi \approx \infty$, depending on the value of (the real part of) $\mu$. The first is characterized by the ansatz

$$
w(\xi)=a \xi^{\beta}+o\left(|\xi|^{\operatorname{Re}(\beta)}\right), \quad \operatorname{Re}(\beta)<0,
$$

and its insertion in (81) yields

$$
\beta=-\frac{1}{\mu}, \quad a^{\mu}=-\exp (i \pi \mu),
$$

which is consistent with (90a) iff

$$
\operatorname{Re}(\mu)>0 \text {. }
$$

The second is characterized by the ansatz

$$
w(\xi)=1+a \xi^{\beta}+o\left(|\xi|^{\operatorname{Re}(\beta)}\right), \quad \operatorname{Re}(\beta)<0,
$$

and its insertion in (81) yields

$$
\beta=\frac{1}{\mu-1}, \quad a^{\mu-1}=1,
$$

which is consistent with (91a) iff

$$
\operatorname{Re}(\mu)<1 \text {. }
$$

We therefore conclude that there are three possibilities: if $\operatorname{Re}(\mu)>1$, only the first ansatz, (90), is applicable, and it characterizes the nature of the branch point of $w(\xi)$ at $\xi=\infty$; if $\operatorname{Re}(\mu)<0$, only the second ansatz, (91), is applicable, and it characterizes the nature of the branch point of $w(\xi)$ at $\xi=\infty$; while if $0<\operatorname{Re}(\mu)<1$, both ansätze, (90) and (91), are applicable, so both types of branch points occur at $\xi=\infty$.

Next, let us investigate the nature of the singularity at $\xi=0$. It is then easily seen, by an analogous treatment, that two behaviors are possible, as displayed by the following ansätze: either

$$
\begin{aligned}
& w(\xi)=a \xi^{\beta}+o\left(|\xi|^{\operatorname{Re}(\beta)}\right), \quad \operatorname{Re}(\beta)>0, \\
& \beta=-\frac{1}{\mu}, \quad a^{\mu}=-\exp (i \pi \mu),
\end{aligned}
$$


which is applicable iff

$$
\operatorname{Re}(\mu)<0 ;
$$

or

$$
\begin{aligned}
& w(\xi)=1+a \xi^{\beta}+o\left(|\xi|^{\operatorname{Re}(\beta)}\right), \quad \operatorname{Re}(\beta)>0, \\
& \beta=\frac{1}{\mu-1}, \quad a^{\mu-1}=1,
\end{aligned}
$$

which is applicable iff

$$
\operatorname{Re}(\mu)>0 \text {. }
$$

This analysis shows that the function $w(\xi)$ features a branch point at $\xi=0$ the nature of which is characterized by the relevant exponent $\beta$, see (92b) or (93b), whichever is applicable (see (92c) and (93c)). But let us emphasize that there is no branch point at all at $\xi=0$ if neither one of the two inequalities (92c) and (93c) holds, namely if $0<\operatorname{Re}(\mu)<1$.

\subsection{Explicitly solvable cases}

Let us end this Section 5 by noting that the equation (81) for certain rational values of $\mu$ reduces to such a low degree polynomial equation that it can be solved explicitly. In particular, the polynomial equation is of second degree if $\mu=-1,1 / 2$ or 2 ; it is of third degree if $\mu=-2,-1 / 2,1 / 3,2 / 3,3 / 2$, or 3 ; while it is of fourth degree if $\mu=-3,-1 / 3,1 / 4,1 / 2,3 / 4,4 / 3$ or 4 .

The diligent reader might wish to use the corresponding explicit solutions formulas for these cases to verify the validity of the previous discussion.

\section{The physical model}

The solution (80) can also be written, via (75), (18) and (5), directly for the particle coordinates $z_{n}(t)$, to read as follows:

$$
\begin{gathered}
z_{s}(t)=Z \mathrm{e}^{i \omega t}-\frac{2 z_{3}(0)-z_{1}(0)-z_{2}(0)}{6 \sqrt{\mu}}[\eta \exp (-2 i \omega t)+1]^{1 / 2} . \\
\cdot\left(-[\check{w}(t)]^{1 / 2}+(-)^{s}[12 \mu-3 \check{w}(t)]^{1 / 2}\right), \quad s=1,2, \\
z_{3}(t)=Z \mathrm{e}^{i \omega t}-\frac{2 z_{3}(0)-z_{1}(0)-z_{2}(0)}{3 \sqrt{\mu}}[\eta \exp (-2 i \omega t)+1]^{1 / 2}[\check{w}(t)]^{1 / 2},
\end{gathered}
$$

where the constant $\eta$ is given in terms of the initial data by (82d) and we set

$$
\check{w}(t) \equiv w[\xi(t)],
$$

so that this dependent variable is now the solution of the nondifferential equation (see (81))

$$
[\check{w}(t)-1]^{\mu-1}[\check{w}(t)]^{-\mu}=R \exp (2 i \omega t)+\bar{\xi}=R[\exp (2 i \omega t)+\eta],
$$

where the constants $R, \bar{\xi}$ and $\eta$ are defined in terms of the initial data, see (82) (and recall that the initial data $\zeta_{n}(0)$ can be replaced by the initial data $z_{n}(0)$, see (6) ). The dependent variable $\check{w}(t)$ is of course the solution of this equation, (96), identified by continuity, as the time $t$ unfolds from $t=0$, from the initial datum $\check{w}(0)=w_{0}$ assigned 
at $t=0$, see (84): this specification is necessary, since generally the nondifferential equation (81) has more than a single solution, in fact possibly even an infinity of solutions.

A discussion of the behaviour of this solution of the initial-value problem of our model (7) with (2) clearly hinges on ascertaining how the solution $\check{w}(t)$ of (96) evolves in time. This equation (96) corresponds of course to the combination of (81) with (82a). Hence one must firstly elucidate the structure of the Riemann surface defined by the dependence of $w(\xi)$ on the complex variables $\xi$ as determined by the nondifferential equations (81), and then understand the consequences of a travel on this Riemann surface when the complex variable $\xi$ evolves according to (82a), namely it travels round and round on the circle of center $\bar{\xi}$ and radius $|R|$ in the complex $\xi$-plane.

The first task is simple, its foundation being provided by the analysis provided in Sections 5.1 and 5.2

The second task is much more demanding, inasmuch as it hinges on the detailed manner the sheets of the Riemann surface are connected via the cuts associated with the branch points discussed in Sections 5.1 and 5.2. The main results of this analysis have already been reported (without proofs) in 1]; their derivation requires a sufficiently extended treatment to suggest a separate presentation [12. To avoid unnecessary repetitions, also the detailed analysis of the Riemann surface associated to (81) is postponed to [12.

\section{Outlook}

In this paper we report a deeper analysis of the model introduced in [1] explaining many results that were there reported without proof (such as the derivation of the general solution by quadratures) while adding some new material (such as a detailed analysis of the equilibrium configurations, small oscillations and similarity solutions of the model). The novelty of this approach in accounting for a new phenomenology associated to chaotic motion in dynamical systems lies in the fact that the solution is a multi-valued function of (complex) time, and a detailed analysis of its Riemann surface leads to very specific predictions in the simpler ( $\mu$ rational) cases, while it also unveils a source of irregular behaviour in the more complicated ( $\mu$ irrational) cases unpredictable inasmuch as the determination of its evolution requires knowledge with arbitrarily large precision of the initial data. The full analysis of the dynamics of this model - including the geometry of the associated Riemann surface - is postponed to a future publication [12].

The purpose of this series of papers together with other related projects [17, 19, is to go beyond the local analysis performed in the literature relating analytic properties of solutions in complex time with dynamical properties of the model (PainlevéKowalewskaya and its non-meromorphic extensions) and to perform a full description of the global properties of the Riemann surface. This full description requires not just finding the type of branch points and their positions, but specifying how the different sheets of the Riemann surface are attached together at those branch points.

Whenever possible, such an approach provides very detailed information on the dynamics that cannot be obtained by the more classical local analyses. 
Towards a Theory of Chaos Explained as Travel on Riemann Surfaces

\section{Acknowledgments}

We would like to thank the Centro Internacional de Ciencias in Cuernavaca, in particular François Leyvraz and Thomas Seligman, for their support in organizing the Scientific Gatherings on Integrable Systems and the Transition to Chaos which provided several opportunities for us to meet and work together. It is a pleasure to acknowledge illuminating discussions with Boris Dubrovin, Yuri Fedorov, Jean-Pierre Françoise, Peter Grinevich, François Leyvraz, Alexander Mikhailov, Thomas Seligman and Carles Simó.

The research of DGU is supported in part by the Ramón y Cajal program of the Spanish ministry of Science and Technology and by the DGI under grants MTM200600478 and MTM2006-14603.

\section{Appendix A}

In this appendix we solve, in the semisymmetrical case, see (2), the nonlinear algebraic equations (23) that characterize the equilibrium configurations and we thereby compute the "eigenvalue" $\gamma^{(3)}$, namely we obtain its two expressions (36a) and $(36 b)$.

The equations to be solved read (see (23))

$$
\begin{aligned}
& \alpha_{1}=\frac{2 g}{\alpha_{1}-\alpha_{3}}+\frac{2 f}{\alpha_{1}-\alpha_{2}}, \\
& \alpha_{2}=\frac{2 g}{\alpha_{2}-\alpha_{3}}-\frac{2 f}{\alpha_{1}-\alpha_{2}}, \\
& \alpha_{3}=\frac{2 g}{\alpha_{3}-\alpha_{1}}+\frac{2 g}{\alpha_{3}-\alpha_{2}},
\end{aligned}
$$

and they of course imply the relation

$$
\alpha_{1}+\alpha_{2}+\alpha_{3}=0 .
$$

It is now convenient to set

$$
S=\alpha_{1}+\alpha_{2}, \quad D=\alpha_{1}-\alpha_{2},
$$

entailing

$$
\alpha_{1}=\frac{S+D}{2}, \quad \alpha_{2}=\frac{S-D}{2}, \quad \alpha_{3}=-S .
$$

From (the sum of) (97ad) and (97b) we easily get

$$
S\left(9 S^{2}-D^{2}\right)=24 g S,
$$

and from this we get two types of solutions. The first solution is characterized by $S=0$, implying (see (99b) and (97ai))

$$
\alpha_{3}=0, \quad \alpha_{1}=-\alpha_{2}=\alpha, \quad \alpha^{2}=f+2 g,
$$

entailing (via (22)) the solution (34) for the equilibrium configuration, as well as (via (24b) with (21) the expressions

$$
\begin{aligned}
& \beta_{3}=\frac{f}{2(f+2 g)}, \\
& \beta_{1}=\beta_{2}=\frac{2 g}{f+2 g},
\end{aligned}
$$


hence, via (32), the first expression, (36a), for $\gamma^{(3)}$.

The second solution is characterized by

$$
9 S^{2}-D^{2}=24 \mathrm{~g} \text {. }
$$

We now subtract (97b) from (97a) and we thereby easily get

$$
D^{2}=\frac{-8 g D^{2}}{9 S^{2}-D^{2}}+4 f
$$

hence, via the preceding relation,

$$
D^{2}=3 f, \quad S^{2}=\frac{f+8 g}{3} .
$$

And via (24b) with (2) and (29b) this is easily seen to yield

$$
\beta_{1}+\beta_{2}+\beta_{3}=\frac{f+8 g}{6 g}
$$

namely, via (32), the second expression, (36b), of $\gamma^{(3)}$. Note moreover that, in both cases, one gets the relation

$$
\left(\alpha_{1}-\alpha_{2}\right)^{2}+\left(\alpha_{2}-\alpha_{3}\right)^{2}+\left(\alpha_{3}-\alpha_{1}\right)^{2}=6(f+2 g),
$$

as can be easily verified from (101) as well as from (7) with (105).

\section{Appendix B}

In this Appendix we consider certain nongeneric (classes of) solutions of our physical problem (7), characterized by special subclasses of initial data.

If the initial data are such that $\eta$, hence as well $\bar{\xi}$, vanish, $\eta=\bar{\xi}=0$ - and this entails that the initial data satisfy the condition

$$
\left[z_{1}(0)-z_{2}(0)\right]^{2}+\left[z_{2}(0)-z_{3}(0)\right]^{2}+\left[z_{3}(0)-z_{1}(0)\right]^{2}=\frac{3(f+2 g)}{i \omega},
$$

see (82) and (6); hence these initial data are not generic, depending only on 2 arbitrary complex parameters rather than on 3 such parameters (or, equivalently, only on 1 rather than 2 such parameters besides the trivial constant $Z$ that only affects the center-of-mass motion, see (6) $)$ - then the time evolution of the solution $z_{n}(t)$ of our physical problem (7), see (6), is clearly periodic with the period $\frac{T}{q}$ rather than $T$. The consequence of this fact are sufficiently obvious not to require any additional elaboration. An example of this type is that characterized by the parameters

$$
\omega=f=2 \pi, \quad g=\pi, \quad \Rightarrow \quad T=\frac{1}{2}, \quad \mu=\frac{p}{q}=\frac{2}{5},
$$

and the initial data

$$
z 1(0)=0.2, \quad z_{2}(0)=-0.79658+0.71779 \mathrm{i}, \quad z_{3}(0)=0.59658-0.71779 \mathrm{i}
$$

that imply that the center of mass is initially at the origin and therefore stays there for all time, $Z=0$. These initial data are easily seen to satisfy the condition (108). 
Towards a Theory of Chaos Explained as Travel on Riemann Surfaces

\section{Appendix C}

In this Appendix we explain how to integrate the ODE (72) in the general case when the three coupling constants $g_{n}$ are all different, namely when the restriction (2) identifying the semisymmetrical case does not apply, and we also provide the solution of the ODE (72) in the two special cases (belonging to the semisymmetrical class characterized by the restriction (2) the treatment of which had been omitted in Section [5] and as well in another special case not belonging to the semisymmetrical class.

\section{Solution of equation (72) in the general case}

In this subsection of Appendix $\mathrm{C}$ we indicate how the ODE (72) can be integrated in the general case when the three coupling constants $g_{n}$ are all different. It is then convenient to set

$$
V(\tau)=\tan [\theta(\tau)],
$$

so that this ODE reads

$$
\frac{V^{\prime} V\left(V^{2}-3\right)}{\left(V^{2}+1\right)\left(A V^{3}+C V^{2}+A V+C-2\right)}=\frac{1}{\left(\tau-\tau_{1}\right)}
$$

with

$$
A=\frac{\sqrt{3}\left(g_{1}-g_{2}\right)}{2\left(g_{1}+g_{2}+g_{3}\right)}, \quad C=\frac{4 g_{1}+4 g_{2}+g_{3}}{2\left(g_{1}+g_{2}+g_{3}\right)} .
$$

To integrate this ODE we set

$$
A V^{3}+C V^{2}+A V+C-2=A\left(V-V_{1}\right)\left(V-V_{2}\right)\left(V-V_{3}\right),
$$

so that the three quantities $V_{n}$ are the three roots of this polynomial of third degree in $V$. We then decompose this rational function of $V$ in simple fractions,

$$
\frac{V\left(V^{2}-3\right)}{\left(V^{2}+1\right)\left(A V^{3}+C V^{2}+A V+C-2\right)}=\sum_{j=1}^{5} \frac{\mu_{j}}{V-V_{j}},
$$

where of course

$$
V_{4}=i, \quad V_{5}=-i,
$$

and the five quantities $\mu_{j}$ are easily evaluated in terms of the 3 roots $V_{n}$ :

$$
\mu_{j}=V_{j}\left(-3+V_{j}^{2}\right) \prod_{k=1, k \neq j}^{5}\left(V_{j}-V_{k}\right)^{-1}
$$

The integration of the ODE (111) is now trivial (using (114)), and it yields (using (115) ) the final formula

$$
[V(\tau)-i]^{\mu_{4}}[V(\tau)+i]^{\mu_{5}} \prod_{n=1}^{3}\left[V(\tau)-V_{n}\right]^{\mu_{n}}=K\left(\tau-\tau_{1}\right),
$$

where $K$ is the integration constant. 
Solution of equation (72) in two special subcases of the semisymmetrical case

In this subsection of Appendix $\mathrm{C}$ we provide the solution of the ODE (72) in the two special subcases (of the semisymmetrical case) the treatment of which had been omitted in Section [5, and as well in another special case not belonging to the semisymmetrical class. If

$$
g_{1}+g_{2}+g_{3}=0
$$

$\rho$ is constant (namely $\tau$-independent, $\rho(\tau)=\rho(0)$, see (69c)). Moreover, via the restriction (2) characterizing the semisymmetrical class, we get (see also (14))

$$
f=-2 g, \quad \mu=0 .
$$

Then (74) is replaced by

$$
u(\tau) \exp \left[-2 u^{2}(\tau)\right]=\exp \left[\frac{3 f\left(\tau-\tau_{0}\right)}{\rho^{2}(0)}\right] .
$$

Let us also note that, if (2) were replaced by

$$
g_{1}=-g_{2}=g, \quad g_{3}=0,
$$

which is also consistent with (118), then (74) with (71) would be replaced by

$$
\theta(\tau)+\sin [2 \theta(\tau)]=\frac{2 \sqrt{3} g\left(\tau_{0}-\tau\right)}{\rho^{2}(0)} .
$$

Returning to the semisymmetrical case characterized by validity of the restriction (2) we now consider the second case whose treatment had been omitted in Section 5 . namely

$$
f=-8 g .
$$

Note that in this case $\mu$ diverges, see (14). Then (74) is replaced by

$$
u(\tau) \exp \left[u^{2}(\tau)\right]=\left[K\left(\tau-\tau_{1}\right)\right]^{-1 / 2} .
$$

\section{Appendix D: relation with more standard (Newtonian) three-body problems}

In this Appendix we indicate the relation among the three-body problems treated in this paper, characterized by equations of motion of Aristotelian type ("the particle velocities are proportional to assigned external and interparticle forces"), with analogous many-body problems characterized by equations of motion of Newtonian type ("the particle accelerations are proportional to assigned external and interparticle forces"). The results reviewed in this section are of interest inasmuch as they relate the model treated in this paper to other, somewhat more physical and certainly more classical, many-body problems, including a prototypical three-body model introduced, and shown to be solvable by quadratures, by Carl Jacobi one and a half centuries ago 21], and the one-dimensional Newtonian many-body problem with two-body forces proportional to the inverse cube of the interparticle distance introduced and solved over four decades ago (firstly in the quantal context [5, 6] and then in the classical context [25, 26]), which contributed to the bloom in the investigation of integrable dynamical systems of the last few decades (see for instance [27, 8]). 
By differentiating the equations of motion (11) and using them again to eliminate the first derivatives in the right-hand sides one gets the following second-order equations of motion of Newtonian type:

$$
\begin{aligned}
\zeta_{n}^{\prime \prime}= & -\frac{2 g_{n+1}^{2}}{\left(\zeta_{n}-\zeta_{n+2}\right)^{3}}-\frac{2 g_{n+2}^{2}}{\left(\zeta_{n}-\zeta_{n+1}\right)^{3}} \\
& +\frac{g_{n+1}\left(g_{n}-g_{n+2}\right)}{\left(\zeta_{n}-\zeta_{n+2}\right)^{2}\left(\zeta_{n+2}-\zeta_{n+1}\right)}+\frac{g_{n+2}\left(g_{n}-g_{n+1}\right)}{\left(\zeta_{n}-\zeta_{n+1}\right)^{2}\left(\zeta_{n+1}-\zeta_{n+2}\right)} .
\end{aligned}
$$

Likewise from the equations of motion (17) one gets

$$
\begin{aligned}
\ddot{z}_{n}+\omega^{2} z_{n}= & -\frac{2 g_{n+1}^{2}}{\left(z_{n}-z_{n+2}\right)^{3}}-\frac{2 g_{n+2}^{2}}{\left(z_{n}-z_{n+1}\right)^{3}} \\
& +\frac{g_{n+1}\left(g_{n}-g_{n+2}\right)}{\left(z_{n}-z_{n+2}\right)^{2}\left(z_{n+2}-z_{n+1}\right)}+\frac{g_{n+2}\left(g_{n}-g_{n+1}\right)}{\left(z_{n}-z_{n+1}\right)^{2}\left(z_{n+1}-z_{n+2}\right)} .
\end{aligned}
$$

Of course the solutions of the first-order equations of motion, (10) respectively (7), satisfy as well the corresponding second-order equations of motion, (123) respectively (124), but they provide only a subset of the solutions of the latter. On the other hand it is again true that the solutions of the second-order equations of motion (123) and (124) are related via the trick.

In the integrable "equal-particle" case, see (3), these equations of motion simplify and correspond respectively to the Newtonian equations of motion yielded by the two standard $N$-body Hamiltonians

$$
H(\underline{\zeta}, \underline{\pi})=\sum_{n=1}^{N} \frac{\pi_{n}^{2}}{2}-\sum_{m, n=1 ; m \neq n}^{N} \frac{g^{2}}{2\left(\zeta_{n}-\zeta_{m}\right)^{2}},
$$

respectively

$$
H(\underline{z}, \underline{p})=\sum_{n=1}^{N} \frac{p_{n}^{2}+\omega^{2} z_{n}^{2}}{2}-\sum_{m, n=1 ; m \neq n}^{N} \frac{g^{2}}{2\left(z_{n}-z_{m}\right)^{2}},
$$

with $N=3$, the complete integrability of which is by now a classical result (even in the $N$-body case with $N>3$ : see for instance [8]).

In fact the more general three-body Hamiltonian models

$$
H(\underline{\zeta}, \underline{\pi})=\sum_{n=1}^{3}\left[\frac{\pi_{n}^{2}}{2}-\frac{g_{n}^{2}}{\left(\zeta_{n+1}-\zeta_{n+2}\right)^{2}}\right],
$$

respectively

$$
H(\underline{z}, \underline{p})=\sum_{n=1}^{3}\left[\frac{p_{n}^{2}+\omega^{2} z_{n}^{2}}{2}-\frac{g_{n}^{2}}{\left(z_{n+1}-z_{n+2}\right)^{2}}\right],
$$

featuring three different coupling constants $g_{n}$, that yield the equations of motion

$$
\zeta_{n}^{\prime \prime}=-\frac{2 g_{n+1}^{2}}{\left(\zeta_{n}-\zeta_{n+2}\right)^{3}}-\frac{2 g_{n+2}^{2}}{\left(\zeta_{n}-\zeta_{n+1}\right)^{3}},
$$

respectively

$$
\ddot{z}_{n}+\omega^{2} z_{n}=-\frac{2 g_{n+1}^{2}}{\left(z_{n}-z_{n+2}\right)^{3}}-\frac{2 g_{n+2}^{2}}{\left(z_{n}-z_{n+1}\right)^{3}},
$$


are also solvable by quadratures. For the equations of motion (129) this discovery is due to Carl Jacobi 21; while the solutions of the equations of motion (130) can be easily obtained from those of the equations of motion (129) via the trick (5). For a detailed discussion of these solutions, and additional indications on key contributions to the study of this problem, the interested reader is referred to [13] and 8 . But we will perhaps also revisit this problem, because we believe that additional study of these models, (129) and (130), shall shed additional light on the mechanism responsible for the onset of a certain kind of deterministic chaos, as discussed above.

Finally let us recall that our Aristotelian model (7), as well as the Newtonian models described in this Appendix, describing "particles" moving in the complex zplane, can be easily reformulated as models describing particles moving in the real plane, with rotation-invariant (or at least covariant) real two-vector equations of motion (see for instance Chapter 4 of [8]).

[1] F. Calogero, D. Gomez-Ullate, P. M. Santini and M. Sommacal, On the transition from regular to irregular motions, explained as travel on Riemann surfaces, J. Phys. A: Math. Gen. 38 (2005) 8873-8896 .

[2] T. Bountis, Investigating non-integrability and chaos in complex time, Physica D86 (1995) 256-267.

[3] T. Bountis, L. Drossos, and I. C. Percival. Nonintegrable systems with algebraic singularities in complex time. J. Phys. A (1991) 24 3217-3236.

[4] T. Bountis, H. Segur and F. Vivaldi, Integrable Hamiltonian systems and the Painlevé property, Phys. Rev. A, 25 (1982) 1257-1264.

[5] F. Calogero, Solution of a three-body problem in one dimension, J. Math. Phys 10 (1969) 2191-2196.

[6] F. Calogero, Solution of the One Dimensional $N$-Body Problem with Quadratic and/or Inversely Quadratic Pair Potentials, J. Math. Phys. 12 (1971) 419-436 ; Erratum: J.Math.Phys. 37 (1996) 3646.

[7] F. Calogero, A class of integrable Hamiltonian systems whose solutions are (perhaps) all completely periodic, J. Math. Phys. 38 (1997) 5711-5719.

[8] F. Calogero, Classical many-body problems amenable to exact treatments, Lecture Notes in Physics Monograph m 66, Springer, Berlin 2001.

[9] F. Calogero, Isochronous systems, Oxford University Press, Oxford 2008.

[10] F. Calogero and J.-P. Françoise. Periodic motions galore: how to modify nonlinear evolution equations so that they feature a lot of periodic solutions. J. Nonlinear Math. Phys., 9 (2002) 99-125.

[11] F. Calogero, J.-P. Françoise and M. Sommacal, Periodic solutions of a many-rotator problem in the plane. II. Analysis of various motions, J. Nonlinear Math. Phys. 10 (2003) 157-214.

[12] F. Calogero, D. Gomez-Ullate, P. M. Santini and M. Sommacal, Towards a theory of chaos as travel in Riemann surfaces II, in preparation.

[13] F. Calogero and M. Sommacal, Periodic solutions of a system of complex ODEs. II. Higher periods, J. Nonlinear Math. Phys. 9 (2002) 1-33.

[14] Y. F. Chang and G. Corliss. Ratio-like and recurrence relation tests for convergence of series. J. Inst. Math. Appl. 25 (1980) 349-359.

[15] Y. F. Chang, J. M. Greene, M. Tabor, and J. Weiss. The analytic structure of dynamical systems and self-similar natural boundaries. Physica D 8 (1983) 183-207.

[16] Y. F. Chang, M. Tabor, and J. Weiss. Analytic structure of the Hénon-Heiles Hamiltonian in integrable and nonintegrable regimes. J. Math. Phys. 23 (1982) 531-538.

[17] Yu. Fedorov and D. Gomez-Ullate, A class of dynamical systems whose solutions travel on the Riemann surface of an hyperelliptic function, Physica D 227 (2007) 120-134.

[18] A. S. Fokas and T. Bountis. Order and the ubiquitous occurrence of chaos. Phys. A 228 (1996) 236-244.

[19] P. Grinevich and P. M. Santini, Newtonian dynamics in the plane corresponding to straight and cyclic motions on the hyperelliptic curve $\mu^{2}=v^{n}-1, n \in \mathbb{Z}$ : ergodicity, isochrony, periodicity and fractals, Physica D 232 (2007) 22-32.

[20] E. L. Ince, Ordinary differential equations, Dover, New York, 1956. 
[21] C. Jacobi, Problema trium corporum mutuis attractionibus cubis distantiarum inverse proportionalibus recta linea se moventium, in Gesammelte Werke, vol. 4, Berlin, 1866, pp. 533-539.

[22] M. D. Kruskal and P. A. Clarkson, The Painlevé-Kowalewski and poly-Painlevé tests for integrability, Studies Appl. Math. 86 (1992) 87-165.

[23] M. D. Kruskal, A. Ramani, and B. Grammaticos. Singularity analysis and its relation to complete, partial and nonintegrability. In Partially integrable evolution equations in physics (Les Houches, 1989), volume 310 of NATO Adv. Sci. Inst. Ser. C Math. Phys. Sci., pages 321-372. Kluwer Acad. Publ., Dordrecht, 1990.

[24] G. Levine and M. Tabor. Integrating the nonintegrable: analytic structure of the Lorenz system revisited. Physica D 33 (1988) 189-210.

[25] C. Marchioro, Solution of a three-body scatering problem in one dimension, J. Math. Phys. 11 (1970) 2193-2196.

[26] J. Moser, Three integrable Hamiltonian systems connected with isospectral deformations, Adv. Math. 16 (1975) 197-220.

[27] A. M. Perelomov, Integrable system of classical mechanics and Lie algebras, Birkhäuser, Basel 1990.

[28] A. Ramani, B. Grammaticos, and T. Bountis. The Painlevé property and singularity analysis of integrable and nonintegrable systems. Phys. Rep. 180 (1989) 159-245.

[29] M. Tabor and J. Weiss. Analytic structure of the Lorenz system. Phys. Rev. A 24 (1981) 2157-2167. 